\title{
Active and Passive Direct-Reading Ratio Sets for the Comparison of Audio-Frequency Admittances
}

\author{
Robert D. Cutkosky
}

(July 17, 1964)

\begin{abstract}
Design considerations and constructional details for two audio-frequency direct-reading ratio sets are presented. These devices allow the comparison of admittances with accuracies up to one part in $10^{3}$. The first direct-reading ratio set (DRRS) is based upon operational amplifier circuitry, and utilizes a two-stage amplifier system. With this technique, only a moderate voltage amplification per stage is required, and the circuitry is therefore not difficult to stabilize to prevent oscillation. The second DRRS is purely passive and makes use of two-stage voltage transformers to reduce the detrimental effects of transformer excitation current upon the linearity and ratio stability of the device. Equivalent circuits representing the behavior of critical parts of the two direct-reading ratio sets are presented and analyzed. A convenient procedure for calibrating a DRRS is treated mathematically.
\end{abstract}

\section{Introduction}

For many years direct-reading ratio sets have been used extensively for the most precise comparisons of d-c resistances. The extension of the direct-reading ratio set (DRRS) principle to audio-frequency bridges is a logical step, but one which is, unfortunately, somewhat difficult to achieve. The principle complication results from the necessity of providing the a-c ratio with both in-phase and quadrature adjustments.

Two types of a-c DRRS constructed at NBS are described here. The first type (sec. 2 of this paper) is based upon operational amplifier circuitry. The use of active networks in bridges is not new, and has been seen in various forms in both special purpose and commercial bridges $[1,2] .{ }^{1} \quad$ Our application called for considerably higher accuracy than was attained in the above cited references, and required the development of special circuitry with high effective loop gains.

Experience with the active DRRS indicated that the bridge circuit was more dependent upon oscillator waveform than had been anticipated. It was found that limiting the active circuit input voltage was necessary to secure satisfactory operation. A completely passive DRRS is described in sec. 3 of this paper. The passive device is much more linear and reliable than the active circuit and was constructed to meet our anticipated need for routine admittance comparisons at a fixed frequency, with precisions up to one part in $10^{9}$ for admittances between $10^{-8}$ and $10^{-5}$ mhos.

The actual mathematics of a-c direct-reading ratio sets is merely a complex version of the mathematics associated with their d-c prototypes [3]. An analysis of some calibration problems is included in sec. 4 of this paper.

\section{Active Circuit}

The active networks to be described in this section are all modifications and extensions of the operational amplifier circuit of figure 1. An analysis of this circuit yields the equation

$$
e_{0}=-e_{s} \frac{Y_{1}}{Y_{2}} \times \frac{1}{1+\frac{\left(1+Y_{2} R+Y_{s}^{\prime} R\right)\left(Y_{d}+Y_{1}+Y_{2}\right)}{\left(A-Y_{2} R\right) Y_{2}}} .
$$

If the amplification $A$ is very high, eq (1) reduces to

$$
e_{0} \approx-e_{s} \frac{Y_{1}}{Y_{2}}
$$

1 Figures in brackets indicate the literature references at the end of this paper. 
The desirable features of this network are the following:

(1) The device generates a voltage $e_{0}$ which is proportional to an input $e_{s}$ and depends only slightly upon the active circuit parameters.

(2) The proportionality constant is essentially the ratio of two three-terminal admittances, may be real or complex, and may be made to exhibit a high order of stability.

(3) The circuit input admittance is approximately equal to $Y_{1}+Y_{s}$. If it is made small enough, the source $e_{s}$ will not be strongly loaded.

(4) The circuit output impedance is usually quite low; a calculation yields $Z_{\text {out }} \approx$ $\frac{R\left(Y_{1}+Y_{2}+Y_{d}\right)}{A Y_{2}} \cdot$ Reasonable loads may therefore be driven by the device, with no appreciabla change in $e_{0}$.

The circuit of figure 1 may be modified as in figure 2 to accommodate two inputs. The usual superposition arguments may be used to relate the behavior of this circuit to eqs (1) and (2). The case in which

$$
Y_{2}=Y_{1}=-j Y_{1}^{\prime}
$$

is of particular interest. Then, given sufficiently high amplification,

$$
e_{0}=-e_{s}-j e_{s}^{\prime}
$$

A bridge for comparing two admittances $Y_{3}$ and $Y_{4}$ is shown in figure 3 . The bridge features a phase splitting transformer, $T_{1}$, and two decade transformers to allow operation in all four electrical quadrants. If $K_{1}$ and $K_{2}$ are the decade transformer readings when the bridge is balanced, we find in the limit as $A \rightarrow \infty$

$$
Y_{4}=2 Y_{3}\left[\left(K_{1}-0.5\right)+j\left(K_{2}-0.5\right)\right] .
$$

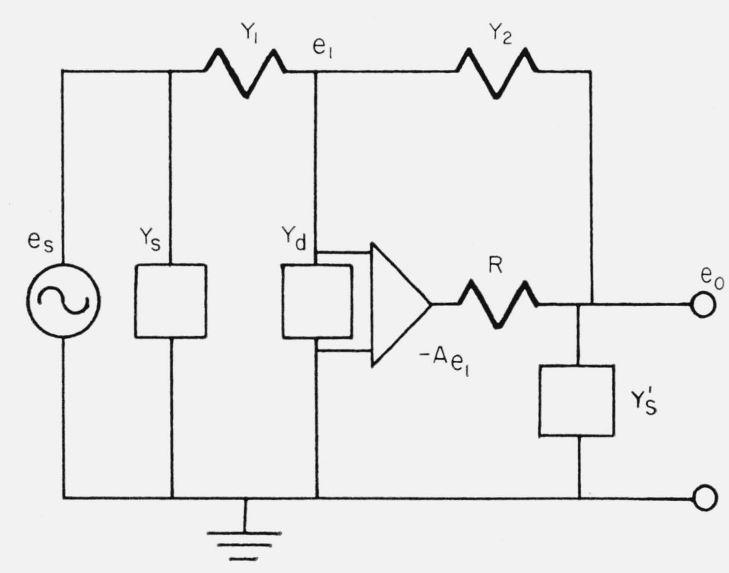

Figure 1. Basic operational amplifier circuit.

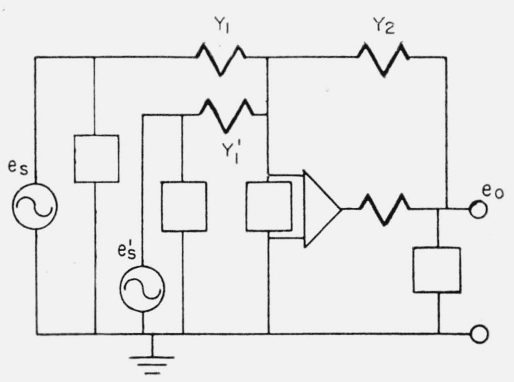

Figure 2. Double-input operational amplifier circuit.

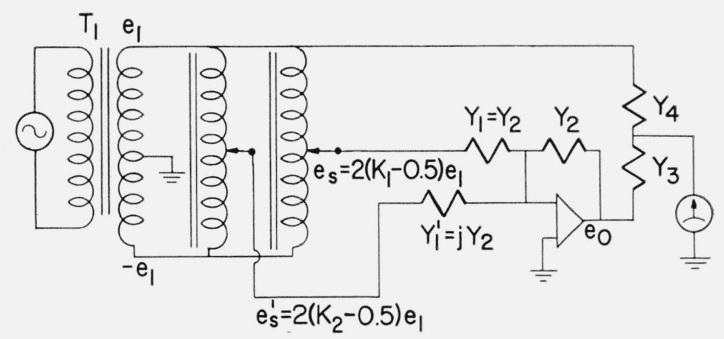

Figure 3. Generalized admittance bridge. 
A DRRS may be produced from the circuit of figure 3 by adding a small fraction of the operational amplifier output voltage, $e_{0}$, to the voltage of a three winding bridge transformer by means of a step-down transformer as in figure 4 . The resultant system behaves like a bridge transformer whose ratio may be varied over a small range by varying $K_{1}$ and $K_{2}$. The stepdown transformer may be a simple two winding transformer, but in this case the voltage ratio will differ from the turns ratio due to primary winding resistance and excitation current. The voltage ratio will then be slightly voltage dependent and unreliable. A better system uses a three winding transformer with the amplifier feedback admittance driven by one of the secondary windings. The other secondary winding then provides a highly accurate voltage which is added to the voltage of the bridge transformer.

Amplifier stability is one of the most difficult problems likely to be encountered in the construction of active systems such as figure 4. In order to make the amplifier output accurate to about one part in $10^{5}$, the amplifier must have a loop voltage amplification of about $10^{5}$, or a power gain of $100 \mathrm{~dB}$. Although this could probably be done with a single amplifier, a two-stage amplifier system, illustrated with a single input in figure 5, was found to be easier to stabilize. A calculation indicates that with the two-stage circuit, the first amplifier of amplification $A_{1}$ produces an output $e_{1}$ with a proportional error in the order of $\left(1+\frac{1}{A_{1}}\right)$. This is corrected by the second amplifier of amplification $A_{2}$ to yield an output with a proportional error in the order of $\left(1+\frac{1}{A_{1} A_{2}}\right)$. Roughly, then, one may make use of two amplifiers with voltage amplification of 300 to replace one amplifier with a voltage amplification of 100,000.

The final version of the circuit in use at NBS is drawn in figure 6. Photographs of the completed unit appear in figures 7 and 8 . Some difficulty was experienced in stabilizing the circuit for all seven tuned frequencies, but these problems were solved using conventional techniques. The circuit is in no sense to be considered an optimum design, but merely as one way of making the system work. The critical admittances are maintained in a constant temperature enclosure and are individually adjusted with suitable shunt, series, or " $T$ " networks using the criteria of section 4. These networks may take the form of figure 9.

The circuit of figure 6 has been in use for about two years, principally at 1592 hertz. It has been found to be remarkably stable, and has required no maintenance other than replacement of tubes. It has proved reliable to a part in $10^{5}$ provided that the input voltage does not exceed $0.2 \mathrm{~V}$ rms. If higher voltages are applied to the amplifier input, the bridge balance may depend upon oscillator harmonic content, the active device filtering action, and detector or active network nonlinearity. These limitations greatly restrict the usefulness of the device, but could possibly be reduced by redesigning the amplifiers. The existing circuit has proved to be highly useful for development work requiring a wide range but only low accuracy; and when used in the DRRS circuit, has been proved reliable to an accuracy of 1 part in $10^{9}$.

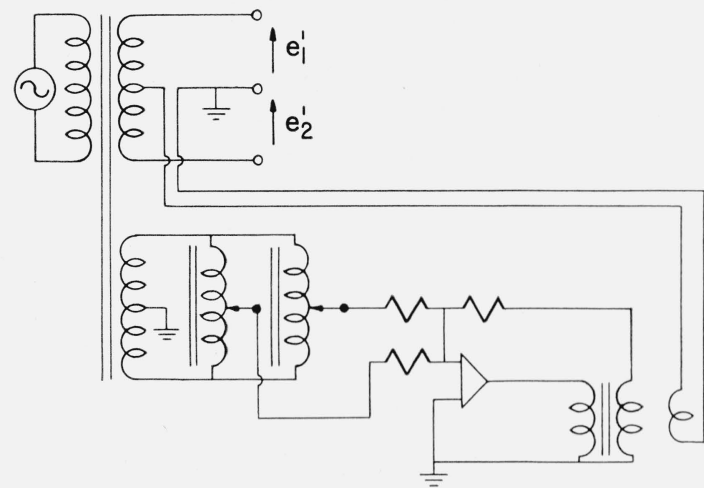

FiguRe 4. Active direct reading ratio set.

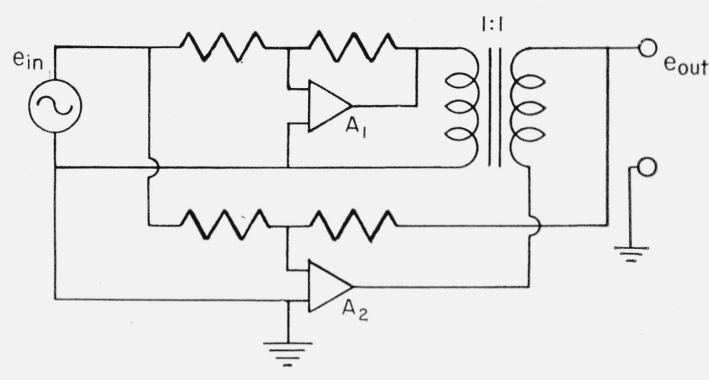

FiguRE 5. Double operational amplifier circuit. 

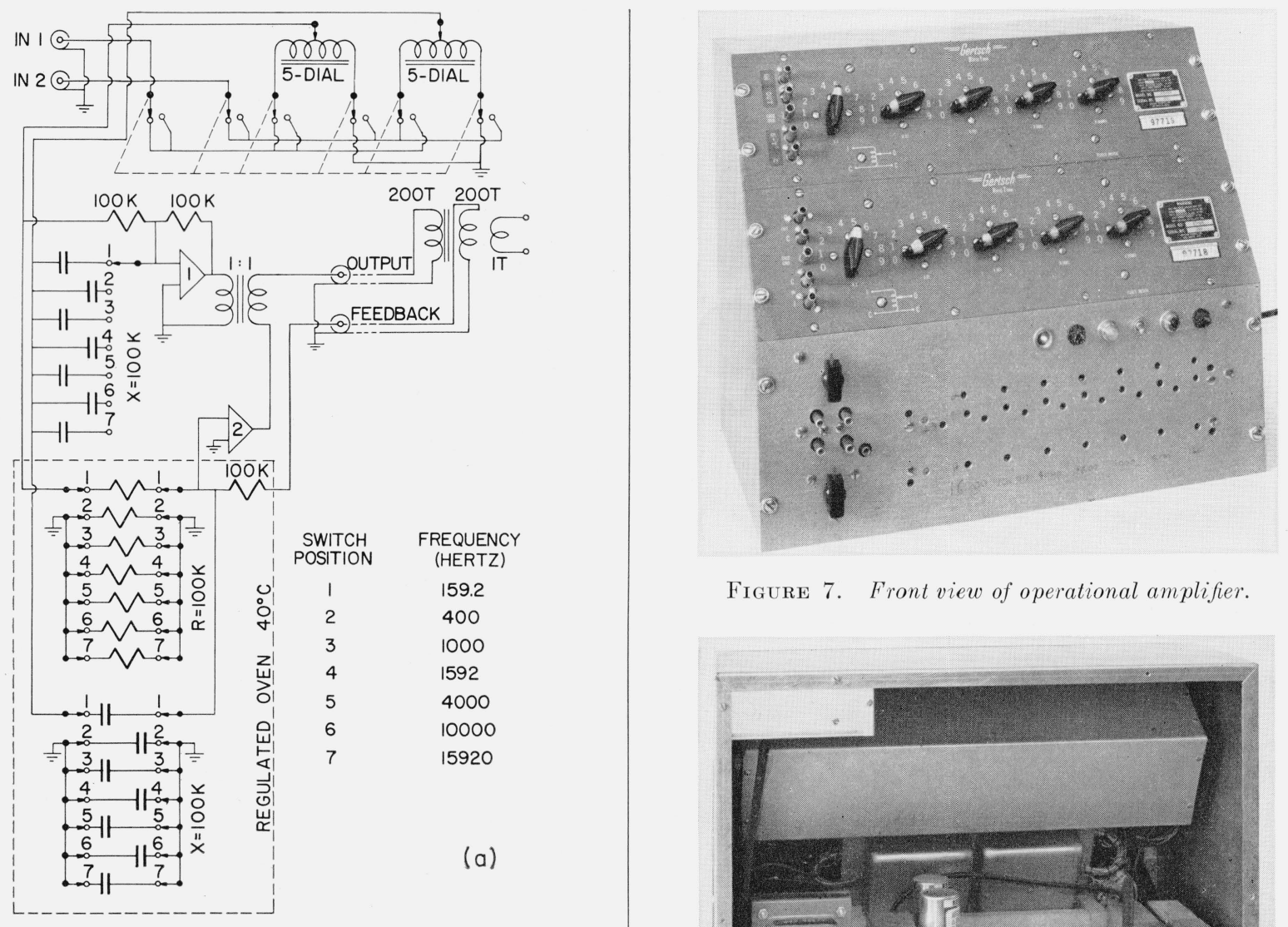

Figure 7. Front view of operational amplifier.
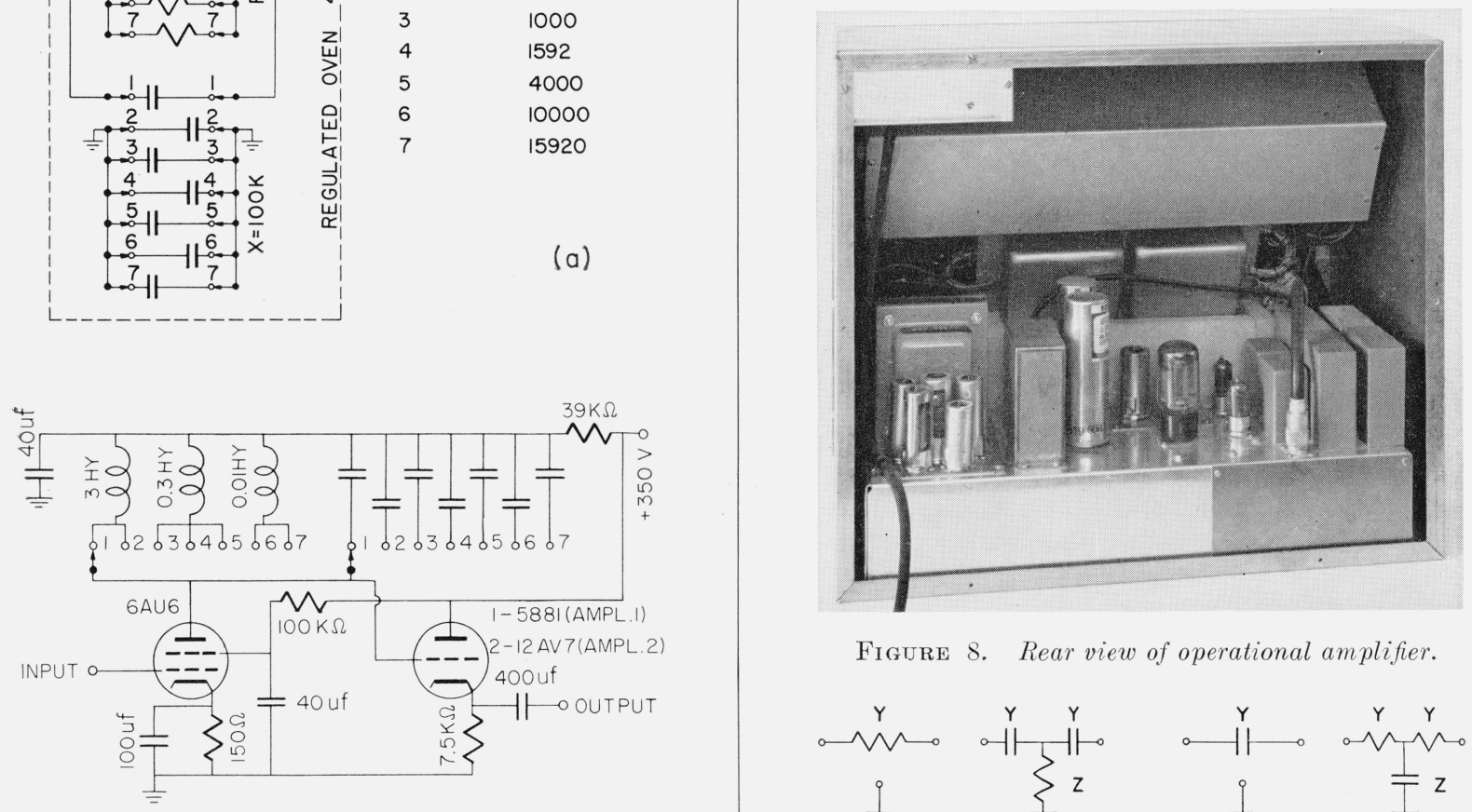

Figine 8. Rear view of operational amplifier.

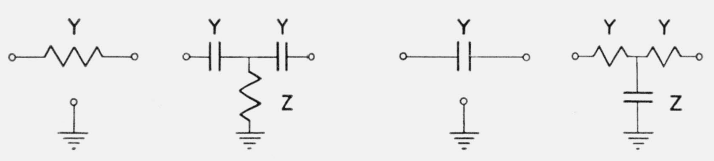

Figure 9. Typical admittance trimmer circuits.

From left to right: positive real, negative real, positive imaginary, and negative imaginary. Valid for $|\mathrm{YZ}|<<1$ where applicable.

\section{Passive Circuit}

All of the functions performed by operational amplifiers in the previous section could have been performed manually by the use of a null detector in place of the amplifier input, and with an adjustable voltage source phase locked with the bridge oscillator in place of the amplifier output. A bridge based on such a system would be awkward to use, since it would require that simultaneous balances be obtained on two null detectors. The situation would be operationally similar to balancing a Schering bridge with a Wagner circuit. 
Bridges with multiple balances are at times extremely useful, and can provide accuracies greater than can be obtained in any other way; but if they can be avoided, a great saving in time results. Since one of the prime advantages of direct-reading ratio sets is speed and ease of operation, a doubly balanced system defeats the whole purpose of the instrument.

Although operational amplifiers can provide a convenient alternative to doubly balanced bridges, most of the workers in precision electrical measurements cultivate a healthy disrespect for all active devices, including even null detectors. The drawbacks of active circuits are many and fairly obvious. They include limited dynamic range without distortion, susceptibility to failure with or without a visible sign of having failed, the inconvenience of having to supply power to the system, and the constructional difficulties of stabilizing a high gain feedback loop.

All of these reasons sugyested that a passive DRRS requiring no auxiliary balances would be a most desirable instrument provided that it could be made stable and accurate. A preliminary investigation disclosed that, although a sufficiently accurate in-phase voltage ratio adjustment could be built, some trouble was to be expected with the phase angle adjustment.

One of the passive circuits investigated and eventually discarded is illustrated in figure 10.

It was found that a combination of factors involving power dissipation in the resistors, nonlinearities in the various transformers, and the dependence of the decade transformer input and output impedances upon dial setting would have restricted the quadrature adjustment accuracy to about one part in $10^{3}$ using readily available components. Since an overall least count of one part in $10^{9}$ was required, the total phase angle adjustment could not have been greater than one part in $10^{6}$, which was judged to be insufficient.

A technique involving a double circuit, one to provide a rough voltage and the other to correct it, was found to be capable of providing the desired range and accuracy. Prominent in the design is a device which may be called a two-stage voltage transformer by virtue of its similarity with the two-stage current transformer [4]. An understanding of two stage voltage transformers is necessary for the understanding of the complete DRRS, and will be dealt with first.

A generalized two stage transformer is represented in figure 11, based on ideal transformers and the usual transformer equivalent circuits [5].

A calculation yields

$$
N e_{3}=\frac{e_{2}\left(1+\frac{z_{1}}{Z}+\frac{z_{1}}{Z^{\prime}}+\frac{z_{2}+z_{2}^{\prime}}{Z^{\prime}}\right)-\frac{z_{2}+z_{2}^{\prime}}{Z^{\prime}}\left(e_{2}-e_{1}\right)}{1+\frac{z_{1}}{Z}+\frac{z_{1}}{Z^{\prime}}+\frac{z_{2}+z_{2}^{\prime}}{Z^{\prime}}+\frac{z_{1}\left(z_{2}+z_{2}^{\prime}\right)}{Z Z^{\prime}}} .
$$

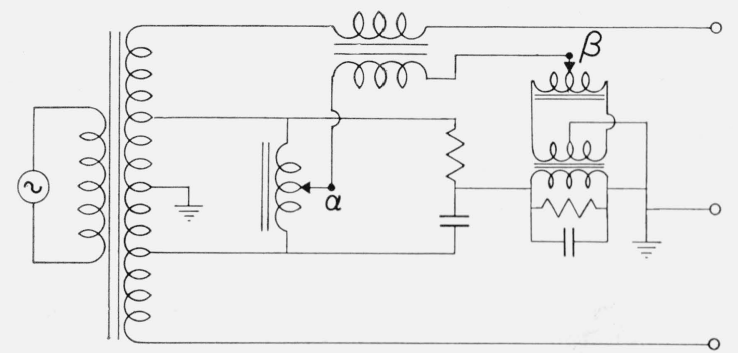

FiguRE 10. Simple passive direct reading ratio set: $\alpha=$ in-phase adjustment, $\beta=$ quadrature adjustment.

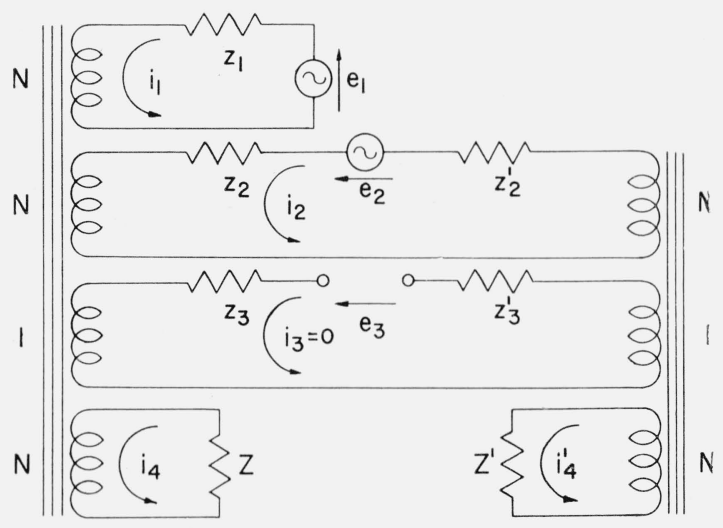

Figure 11. Two stage voltage transformer equivalent circuit. 
If we let $e_{2}-e_{1}=\Delta e$, and neglect terms in the order of $\left(\frac{z}{Z}\right)^{3}, \Delta e\left(\frac{z}{Z}\right)^{2}$, and higher, we have

$$
N e_{3} \approx e_{2}\left[1-\frac{z_{1}\left(z_{2}+z_{2}^{\prime}\right)}{Z Z^{\prime}}\right]-\frac{z_{2}+z_{2}^{\prime}}{Z^{\prime}} \Delta e
$$

It follows that if one uses a common generator, so that $\Delta e=0$, the device acts like a two winding transformer with a highly accurate primary to secondary ratio. Since the ratio of an ordinary transformer varies as $\left(1-\frac{z}{Z}\right)$, quite large improvements can be expected.

Equation 7 also indicates that provided $\Delta e$ is sufficiently small, $e_{3}$ is determined principally by $e_{2}$. A detailed analysis shows in addition that under no-load conditions, appreciably more current flows from generator $e_{1}$ than from generator $e_{2}$, leading to the conclusion that $e_{1}$ may be treated as a "rough" generator to supply excitation current to the two-stage transformer and to obtain approximately the desired output voltage. Generator $e_{2}$ then corrects this approximate voltage, and is itself only slightly loaded in the process. The two-stage transformer of figure 11 can be conveniently constructed as a composite unit, as indicated in figure 12a. This circuit will be represented in what follows by figure $12 \mathrm{~b}$.

The complete passive DRRS, figure 13, is a double version of the circuit of figure 10 .
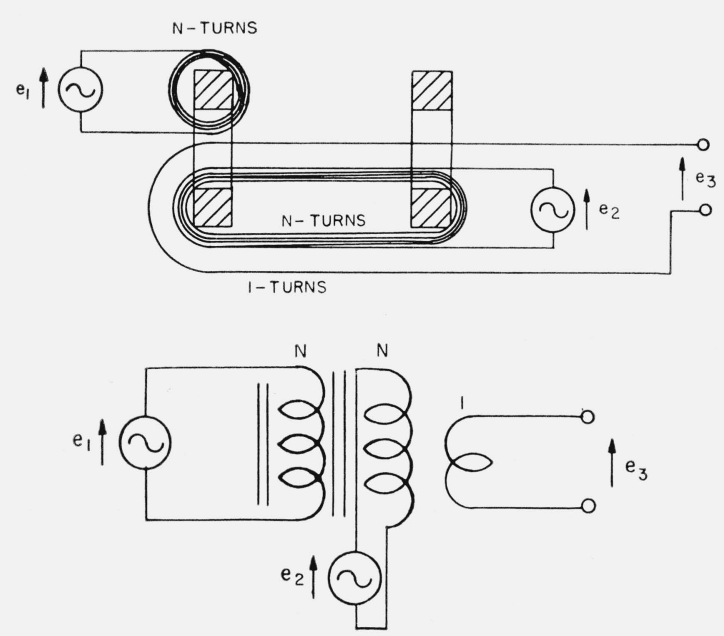

Figure 12. Two stage voltage transformer.

(a) Pictorial representative. (b) Circuit symbol.

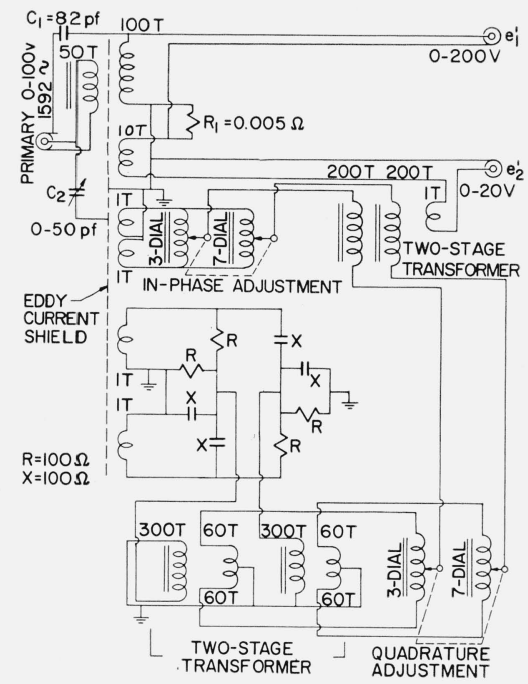

FiguRe 13. Practical passive direct reading ratio set.

The operation of this final version follows from the preceding discussion. The circuit was designed to yield an in-phase voltage ratio variation of $\pm 500 \mathrm{ppm}$ and a phase angle variation of $\pm 50 \mu$ radians. The overall voltage ratio accuracy, after the application of small corrections, was desired to be at least one part in $10^{\circ}$.

The double system is applied in figure 13 to a nominally 10:1 transformer at a single fixed frequency, $1592 \mathrm{~Hz}\left(\omega=10^{4}\right)$. Two views of the completed instrument appear in figures 14 and 15.

Each of the double decade inductive dividers appearing in figure 13 is a commercial 7-dial inductive divider modified by the addition of a 3-dial divider ganged to the first three switches. The added 3-dial divider is constructed with one small toroidal Supermalloy core, and need be no more accurate than 0.1 percent.

The resistors and capacitors of the "rough" and "good" quadrature sources are maintained 


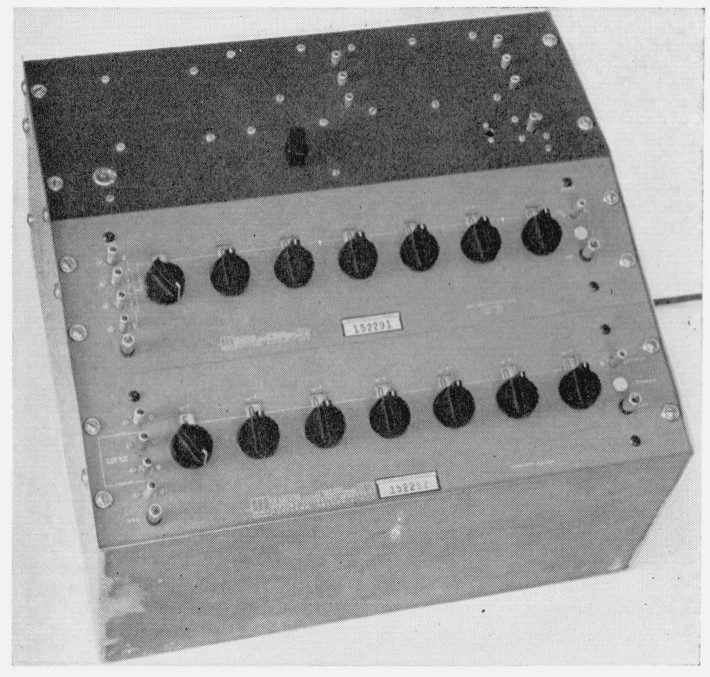

Figure 14. Top view of passive direct reading ratio set.
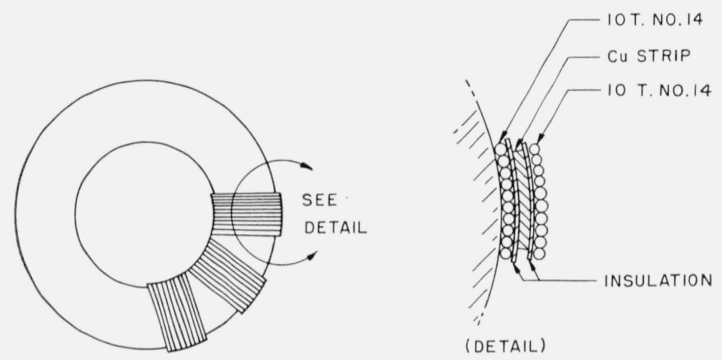

Figure 16. Passive direct reading ratio set main transformer.

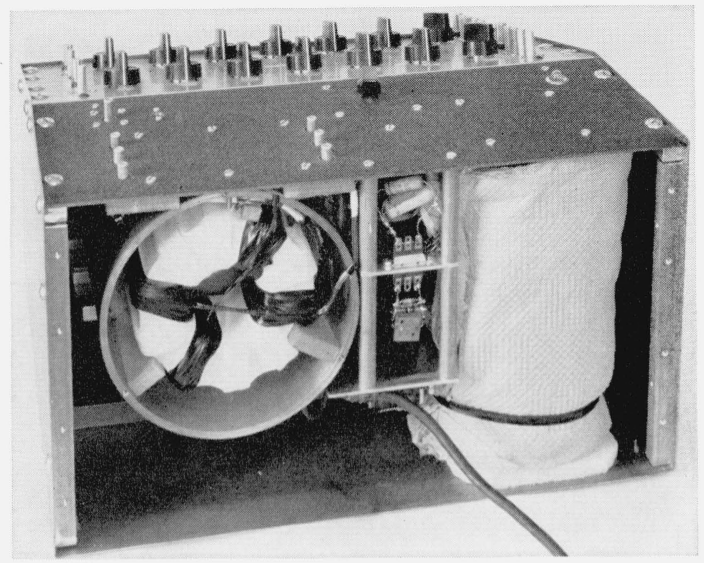

Figure 15. Rear view of passive direct reading ratio set with cover removed.

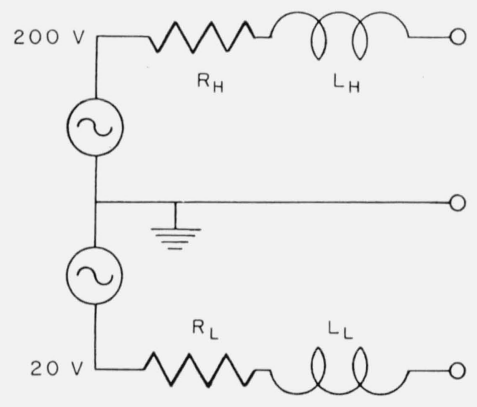

Figure 17. Direct reading ratio set equivalent series impedances.

in a constant temperature enclosure and are of a quality sufficient to ensure that the quadrature part of $e_{3}$ is stable to better than one part in $10^{5}$. The magnitude and phase angle of $e_{3}$ are adjusted by trimming the appropriate resistors and capacitors of the networks with parallel combinations of small capacitors and large resistors.

The main transformer in figure 13 is constructed on a toroidal Supermalloy core of dimensions 4.5 in. o.d. x 3.25 in. i.d. x 1.5 in. high. A copper eddy current shield two skin depths ( $1 / 8$ in.) thick between the primary and secondary windings reduces the magnetic fields in the region of the secondary windings caused by excitation current in the primary winding, and hence improves the accuracy of the secondary ratio.

The 100 turn secondary winding is constructed by first winding 100 turns of \#14 wire as indicated in figure 16. A strip of copper is then wound directly over this to provide the 10 turn secondary winding. Following this, another 100 turns of \#14 wire are wound, and connected in parallel with the first.

A measurement of equivalent series impedances for this transformer yields the circuit of figure 17 and the associated table 1 . The relatively high resistance $R_{H}$ is reduced by use of the resistor $R_{1}$ in figure 13. Although $R_{H}$ could be easily made equal to zero or even negative by increasing $R_{1}$, this would be at the expense of increasing $R_{L}$. The choice of $0.005 \mathrm{ohm}$ for $R_{1}$ represents a compromise based on the intended use for the DRRS. 
TABLE 1. The dependence of the DRRS output impedances upon $\mathrm{R}_{1}$ (see fig. 17)

\begin{tabular}{|c|c|}
\hline$R_{1}=0$ & $R_{1}=0.005 \mathrm{ohm}$ \\
\hline $\begin{array}{ll}R_{H} & 0.085 \\
L_{H} & -2 \times 10^{-6} \\
R_{L} & 0.005 \\
L_{L} & +2 \times 10^{-7}\end{array}$ & $\begin{array}{l}0.035 \mathrm{ohm} \\
-2 \times 10^{-6} \mathrm{H} \\
0.010 \mathrm{ohm} \\
+2 \times 10^{-7} \mathrm{H}\end{array}$ \\
\hline
\end{tabular}

A final feature of the DRRS worthy of comment is the use of capacitors $C_{1}$ and $C_{2}$ to balance out the capacitance from the primary winding to the shield. The capacitive current that would otherwise flow through this capacitance could lead to the net flow of current from the oscillat or to the bridge, and to ground through other paths. Such noncoaxial current paths are usually avoided by using two shields between primary and secondary transformer windings.

\section{Calibration Procedures}

The direct-reading ratio sets described above may be functionally represented by figure 18 . Figure 18a may be called "common lead insertion," since the variable voltage $e_{3}$ appears in series with the main generators $e_{1}$ and $e_{2}$. This system was used with the active DRRS. Figure 18b may be called "single lead insertion," and was used with the passive DRRS. We have from the figure the relationships

and

$$
\frac{e_{1}^{\prime}}{e_{2}^{\prime}}=\frac{e_{1}+e_{3}}{e_{2}-e_{3}} \text { for common lead insertion, }
$$

$$
\frac{e_{1}^{\prime}}{e_{2}^{\prime}}=\frac{e_{1}}{e_{2}+e_{3}} \text { for single lead insertion. }
$$

If the instruments behaved ideally, we could write in both cases

$$
e_{3}=e_{2}\left[M_{1}\left(K_{1}-0.5\right)+j M_{2}\left(K_{2}-0.5\right)\right]
$$

where $K_{1}$ and $K_{2}$ are the readings of the "real" and "imaginary" inductive dividers, and $M_{1}$ and $M_{2}$ are the appropriate scale factors. The terms $\left(K_{1}-0.5\right)$ and $\left(K_{2}-0.5\right)$ occur because the circuit wiring makes $e_{3}=0$ when the inductive dividers are in the middle of their ranges, or read 0.5. Inserting eq (10) into eqs (8) and (9), we have

$$
\frac{e_{1}^{\prime}}{e_{2}^{\prime}}=\frac{e_{1}}{e_{2}} \times \frac{1+\frac{e_{2}}{e_{1}}\left[M_{1}\left(K_{1}-0.5\right)+j M_{2}\left(K_{2}-0.5\right)\right]}{1-\left[M_{1}\left(K_{1}-0.5\right)+j M_{2}\left(K_{2}-0.5\right)\right]}
$$

for common lead insertion and

for single lead insertion.

$$
\frac{e_{1}^{\prime}}{e_{2}^{\prime}}=\frac{e_{1}}{\epsilon_{2}} \times \frac{1}{1+M_{1}\left(K_{1}-0.5\right)+j M_{2}\left(K_{2}-0.5\right)}
$$

The first form is rather peculiar because of the second order dependence of $\frac{e_{1}^{\prime}}{e_{2}^{\prime}}$ upon the $K$ 's. The principal advantage of the common lead insertion method seems to be in the construction of multiple bridges involving more than two equivalent generators, and in 1:1 bridges $\left(e_{1} \approx e_{2}\right)$ for which the resulting symmetrical form of equation 1 may be desirable. The discussion to follow will center around the single lead insertion technique because of its simplicity and its similarity to d-c direct-reading ratio sets. Most of the results may be readily adapted to the common lead insertion technique.

A great many possible deviations from the ideal eq (12) are possible. A strict and unambiguous calibration would require that $e_{2}^{\prime} / e_{1}^{\prime}$ be measured for every possible combina- 

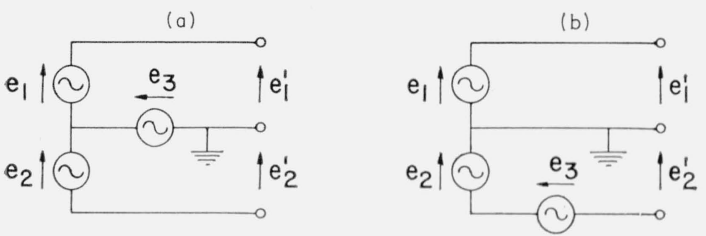

FIguRe 18. Direct reading ratio set functional representation.

(a) Common lead insertion.

(b) Single lead insertion.

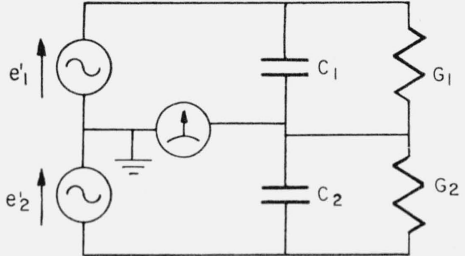

Figure 19. Bridge circuit.

tion of dial settings. A less time-consuming approach is to assume initially that eq (12) is "almost" true, and then to hunt for small discrepancies as $K_{1}$ and $K_{2}$ are systematically varied. Certain errors may conceivably remain undetected under these conditions, but if care is taken to investigate many combinations of dial settings the likelihood will be quite small.

Consider the bridge circuit of figure 19, for which the balance condition is

$$
\frac{e_{2}^{\prime}}{e_{1}^{\prime}}=\frac{j \omega C_{1}+G_{1}}{j \omega C_{2}+G_{2}} \equiv \frac{Y_{1}}{Y_{2}}
$$

Inserting eq (9) we obtain $\frac{Y_{1}}{Y_{2}}=\frac{e_{2}+e_{3}}{e_{1}}$. If $Y_{1}$ is changed by $\Delta Y_{1}$, then $\frac{\Delta Y_{1}}{Y_{2}}=\frac{\Delta e_{3}}{e_{1}}$. By increasing $\Delta Y_{1}$ in known increments we may determine whether or not $e_{3}$ is a linear function of $K_{1}$ and $K_{2}$. In practice it may be convenient to insert first a group of equal capacitances in parallel with $C_{1}$, and then to insert a group of equal conductances.

If $e_{3}$ is indeed a linear function of $K_{1}$ and $K_{2}$, then eq (12) follows immediately; but $M_{1}$ and $M_{2}$ may be complex. If $e_{3}$ is nonlinear, then special techniques must be developed to account for the nonlinearity. The nonlinearity may be of simple form; for example, it may have a second power dependence upon $K_{1}$ and $K_{2}$, or the nonlinearity may be describable with an additive correction to the first dials of the inductive dividers.

Let us assume that the instrument has passed the linearity test, so that eq (12) holds. Then if $M_{1}=m_{11}+j m_{12}$ and $M_{2}=m_{22}+j m_{21}$ where the lower case $m$ 's are real, $N$ equal to the nominal ratio, and finally, if $K_{1}^{\circ}$ and $K_{2}^{\circ}$ are the values of $K_{1}$ and $K_{2}$ for which $\frac{e_{2}^{\prime}}{e_{1}^{\prime}}=N$, we may expand eq (12) to yield

$$
\left.\begin{array}{l}
\frac{e_{2}^{\prime}}{e_{1}^{\prime}}=N \frac{1+M_{1}\left(K_{1}-0.5\right)+j M_{2}\left(K_{2}-0.5\right)}{1+M_{1}\left(K_{1}^{\circ}-0.5\right)+j M_{2}\left(K_{1}^{\circ}-0.5\right)} \\
\begin{array}{l}
e_{2}^{\prime} \\
e_{1}^{\prime}
\end{array} \\
\frac{e_{2}^{\prime}}{e_{1}^{\prime}} \approx N\left[1+M_{1}\left(K_{1}-K_{1}^{\circ}\right)+j M_{2}\left(K_{2}-K_{2}^{\circ}\right)\right] \\
\}\left(m_{11}+j m_{12}\right)\left(K_{1}-K_{1}^{\circ}\right)+j\left(m_{22}+j m_{21}\right)\left(K_{2}-K\right)_{2}^{\circ}\right]
\end{array}\right\}
$$

The terms $K_{1}^{\circ}$ and $K_{2}^{\circ}$ are the components of the complex "zero" reading so familiar to users of d-c direct-reading ratio sets. They need not be known if the instrument is used only for direct substitution measurements, but must be measured, for example, if the DRRS is used to step up from small to large admittances. Methods for measuring $K_{1}^{\circ}$ and $K_{2}^{\circ}$ directly may be obtained by slightly modifying the results of a previous paper [6].

The determination of $m_{11}, m_{12}, m_{21}, m_{22}$ may be performed with the aid of eqs (13) and (14). Balancing the bridge initially as in figure 19 , we write

$$
\frac{j \omega C_{1}+G_{1}}{j \omega C_{2}+G_{2}}=N\left[1+\left(m_{11}+j m_{12}\right)\left(K_{1}-K_{1}^{\circ}\right)+j\left(m_{22}+j m_{21}\right)\left(K_{2}-K_{2}^{\circ}\right)\right] .
$$


Adding in succession two admittances, first $j \omega \Delta C_{1}^{\prime}+\Delta G_{1}^{\prime}$ and then $j \omega \Delta C_{1}^{\prime \prime}+\Delta G_{1}^{\prime \prime}$, we have, upon subtracting eq (15),

and

$$
\left.\begin{array}{l}
\frac{j \omega \Delta C_{1}^{\prime}+\Delta G_{1}^{\prime}}{j \omega C_{2}+G_{2}}=N\left[\left(m_{11}+j m_{12}\right)\left(K_{1}^{\prime}-K_{1}\right)+j\left(m_{22}+j m_{21}\right)\left(K_{2}^{\prime}-K_{2}\right)\right] \\
\frac{j \omega \Delta C_{1}^{\prime \prime}+\Delta G_{1}^{\prime \prime}}{j \omega C_{2}+G_{2}}=N\left[\left(m_{11}+j m_{12}\right)\left(K_{1}^{\prime \prime}-K_{1}\right)+j\left(m_{22}+j m_{21}\right)\left(K_{2}^{\prime \prime}-K_{2}\right)\right]
\end{array}\right\},
$$

from which, if $\frac{j \omega \Delta C_{1}^{\prime}+\Delta G_{1}^{\prime}}{j \omega \Delta C_{1}^{\prime \prime}+\Delta G_{1}^{\prime \prime}}$ is not real, the $m^{\prime}$ s may be determined.

It is expected that normally $G_{2}<<\omega C_{2}, \Delta G_{1}^{\prime}<<\omega \Delta C_{1}^{\prime}, \Delta G_{1}^{\prime \prime}>>\omega \Delta C_{1}^{\prime \prime}, m_{12}<<m_{11}$ and $m_{21}<<m_{22}$, so that

and

$$
\begin{aligned}
& m_{11} \approx \frac{\Delta C_{1}^{\prime}}{N C_{2}^{\prime}} \times \frac{1}{K_{1}^{\prime}-K_{1}}, \\
& m_{12} \approx \frac{\Delta G_{1}^{\prime \prime}\left(K_{2}^{\prime}-K_{2}\right)}{N \omega C_{2}\left(K_{1}^{\prime}-K_{1}\right)\left(K_{2}^{\prime \prime}-K_{2}\right)}-\frac{\Delta G_{1}^{\prime}}{N \omega C_{2}\left(K_{1}^{\prime}-K_{1}\right)}, \\
& m_{22} \approx \frac{-\Delta G_{1}^{\prime \prime}}{N \omega C_{2}} \times \frac{1}{K_{2}^{\prime \prime}-K_{2}}
\end{aligned}
$$

$$
m_{21} \approx \frac{\Delta C_{1}^{\prime}\left(K_{1}^{\prime \prime}-K_{1}\right)}{N C_{2}\left(K_{1}^{\prime}-K_{1}\right)\left(K_{2}^{\prime \prime}-K_{2}\right)}-\frac{\Delta C_{1}^{\prime \prime}}{N C_{2}\left(K_{2}^{\prime \prime}-K_{2}\right)}
$$

The problem is thus reduced to determining the complex ratios of admittances, where only a very modest accuracy is required.

\section{References}

[1] N. L. Kusters and O. Petersons, A Transformer-ratio-arm bridge for high-voltage capacitance measurements, IEEE Trans. on Communications and Electronics, No. 63-168, 69, 606 (1963).

[2] H. P. Hall and R. G. Fulks. The use of active devices in precision bridges, Electrical Engineering $\mathbf{8 1 ,} 367$ (1962).

[3] Paul P. B. Brooks, Calibration procedures for direct-current resistance apparatus, NBS Mono. 39 (1962).

[4] H. B. Brooks and F. C. Holtz, The two-stage current transformer, AIEE Trans. 41, 383 (1922).

[5] A. M. Thompson, The precise measurement of small capacitances, IRE Trans. on Inst. I- $\mathbf{8}$, Nos. 3 and 4 , 245 (1958).

[6] R. D. Cutkosky and J. Q. Shields, The precision measurement of transformer ratios, IRE Trans. on Inst. I-9, No. 2, 243 (1960).

(Paper 68C4-168) 